\title{
On the Quantization of Presymplectic Dynamical Systems via Coisotropic Imbeddings
}

\author{
Mark J. Gotay* and Jędrzej Śniatycki ${ }^{\dagger}$ \\ Department of Mathematics and Statistics, The University of Calgary, \\ Calgary, Alberta, Canada T2N 1N4
}

\begin{abstract}
We study certain aspects of the problem of quantizing a presymplectic dynamical system. Such a system is quantized by imbedding the presymplectic manifold $M$ under consideration into a symplectic manifold $X$ and then geometrically quantizing the latter. It is known that this procedure will yield consistent results provided the imbedding $M \rightarrow X$ is coisotropic; we show that such imbeddings always exist and are "locally unique." Furthermore, we investigate the extent to which the resulting quantum dynamics is independent of the choice of coisotropic imbedding; that is, we examine to what extent the presymplectic phase space $M$ determines the quantum representation space and the quantization of observables. The quantization is carried out within the geometric quantization framework of Kostant and Souriau.
\end{abstract}

\section{Introduction}

Let $(M, \Omega)$ be a presymplectic manifold, that is, a manifold $M$ endowed with a closed 2-form $\Omega$ of constant rank. Such manifolds appear frequently in physics, in which context $M$ represents the (degenerate) phase space of a dynamical system and $\Omega$ generalizes the Lagrange bracket [1]. Indeed, presymplectic phase spaces arise naturally in relativistic dynamics (e.g., spinning particles in curved spacetime [2], Kaluza-Klein electrodynamics [3]), in the Dirac theory of constraints [4-7], in Lagrangian dynamics [5-8], and also in time-dependent dynamics [9-10].

Unfortunately, little is known regarding the quantization of systems described classically by presymplectic geometries, since most quantization procedures have been developed for symplectic systems (i.e., under the assumption that $\Omega$ is nondegenerate). On the other hand, the quantization of symplectic systems is,

\footnotetext{
* Supported in part by the Natural Sciences and Engineering Research Council of Canada through an Operating Grant to The University of Calgary

+ Supported in part by the Natural Sciences and Engineering Research Council of Canada through Operating Grant \#A8091
} 
at this point, relatively well understood [11-13]. Consequently, one approach to quantizing a presymplectic dynamical system is to - in some natural mannerassociate to it a symplectic system and then quantize the latter.

There are many ways of associating a symplectic manifold to a presymplectic manifold $(M, \Omega)$. One method is to "extend" $(M, \Omega)$ to a symplectic phase space. More precisely, one searches for a symplectic manifold $(X, \omega)$ and an imbedding $j: M \rightarrow X$ such that $j^{*} \omega=\Omega$; many such imbeddings can always be found. ${ }^{1}$ Another possibility is to "reduce" $(M, \Omega)$ as follows $[1,14]$. Let $N$ be the characteristic distribution of the presymplectic form $\Omega$, i.e.,

$$
N=\{v \in \mathscr{T} M \mid v\lrcorner \Omega=0\} .
$$

Since $\Omega$ is closed $N$ is involutive; denote by $\bar{M}$ the space of all integral manifolds of $N$. If $\bar{M}$ is a quotient manifold of $M$ with projection $\rho$, then there exists a unique symplectic form $\bar{\Omega}$ on $\bar{M}$ such that $\Omega=\rho^{*} \bar{\Omega}$. The pair $(\bar{M}, \bar{\Omega})$ is called the reduced phase space of $(M, \Omega)$. A third way to associate a symplectic manifold to $(M, \Omega)$ is via the imposition of a gauge condition [15]. This method is particularly appropriate when $M$ is realized as a level set of the momentum mapping defined by the action of a gauge group on some symplectic manifold [16]. Imposing a gauge condition amounts to reducing the gauge group to one of its subgroups. A maximal reduction occurs if the subgroup in question consists only of the identity; this corresponds to giving a section $\bar{M} \rightarrow M$ of the canonical projection $\rho: M \rightarrow \bar{M}$. The image of such a section is a symplectic submanifold of $(M, \Omega)$.

Of the three methods listed above, the first is perhaps the most natural and straightforward. In fact, presymplectic manifolds often arise in practice as submanifolds of symplectic manifolds.This is the case, for instance, in Dirac's theory of constrained classical systems [4-7]. Here, the ambient space $(X, \omega)$ is the phase space of the system under consideration and $M$ is the "constraint set," that is, the submanifold of $X$ consisting of all points which are admissible initial data for the equations of motion of the system. To quantize such a constrained dynamical system, Dirac [17] quantized the ambient space $(X, \omega)$ and then imposed certain constraints on the quantum wave functions. We now briefly review Dirac's procedure, generalizing to the case of an arbitrary presymplectic dynamical system. $^{2}$

Given a presymplectic manifold $(M, \Omega)$, one first imbeds it in some symplectic manifold $(X, \omega)$. Without loss of generality it may be assumed that $M$ is globally defined as a submanifold of $X$ by the vanishing of certain functions ("constraints"). Now, the quantization of the imbedding space $(X, \omega)$ produces a space $\mathscr{H}$ of quantum states and associates to some class of smooth functions $g$ on $X$ quantum operators $2 g$ on $\mathscr{H}$. One then postulates that the physically admissible quantum states of the system are those which belong to the subspace $\mathscr{H}_{0}$ of $\mathscr{H}$ defined by

$$
\mathscr{H}_{0}=\{\sigma \in \mathscr{H} \mid \mathscr{Q} f[\sigma]=0 \text { for all quantizable constraints } f\} .
$$

1 For instance, take $(X, \omega)=\left(\mathscr{T}^{*} M, \omega_{M}+\pi^{*} \Omega\right)$, where $\omega_{M}$ is the canonical symplectic form on $\mathscr{T}^{*} M$ and $\pi: \mathscr{T}^{*} M \rightarrow M$ is the projection. See also Sect. III

2 Examples illustrating Dirac's "quantization via imbedding" program may be found in A. Hanson,

T. Regge and C. Teitelboim, Accademia Nazionale dei Lincei, Rome, 22 (1976) 
In essence, this procedure allows one to quantize an "unconstrained" symplectic system - as opposed to a "constrained" presymplectic system - - by enforcing the constraints on the quantum rather than classical level.

This approach, however, may lead to inconsistencies. Specifically, in the presence of quantizable second class ${ }^{3}$ constraints $\mathscr{H}_{0}$ reduces to zero, i.e., there are no nontrivial eigenstates of the constraint operators $\mathscr{2} f[14,17]$. Hence the method of "quantization via imbedding" may lead to meaningful results only if all constraints are first class. From a global standpoint, this means that in order to obtain consistent quantum dynamics it is necessary to place a restriction on the allowable types of imbeddings $(M, \Omega) \rightarrow(X, \omega)$.

The requirement that all constraints be first class is equivalent to the condition that the imbedding of $(M, \Omega)$ into $(X, \omega)$ be coisotropic [cf. Sect. III]. It is a fundamental fact $[18,19]$ that, for any presymplectic manifold $(M, \Omega)$, such imbeddings always exist and are "locally unique" in the sense that a neighborhood of $M$ in the imbedding space is (up to symplectomorphism) completely determined by $(M, \Omega)$.

These results imply that of the three ways of associating a symplectic manifold to a presymplectic manifold $(M, \Omega)$ listed above, only the first one-leading to an extended phase space-does not require any additional assumptions on $(M, \Omega)$. The method of quantizing a presymplectic dynamical system "via imbedding" is therefore the most generally applicable one, and we will accordingly restrict consideration to it in this paper.

The fact that a neighborhood of the coisotropic submanifold $M$ in the extended phase space $(X, \omega)$ is completely determined by $(M, \Omega)$ up to symplectomorphism is quite significant. Physically, this means that in a local sense it is possible to canonically associate to every presymplectic dynamical system a symplectic system. Furthermore, it implies that the classical symplectic dynamics obtained in this manner is effectively independent of the choice of coisotropic imbedding. This, of course, is to be expected since it is the presymplectic phase space $(M, \Omega)$ that is of primary importance rather than the auxiliary ambient space $(X, \omega)$.

The purpose of this paper is to determine the extent to which this classical property carries over to the quantal domain. That is, to what extent is the quantum dynamics of the system independent of the choice of coisotropic imbedding? To what extent does the coisotropic submanifold $M$ determine the space of quantum states and the quantization of observables? More precisely, we investigate the conditions under which the quantum representation space $\mathscr{H}$ corresponding to the symplectic phase space $(X, \omega)$ can be reconstructed from the knowledge of the values of the wave functions on the submanifold $M$. We also inquire as to whether the knowledge of a classical dynamical variable $f$ in an (arbitrarily small) neighborhood of $M$ in $X$ is sufficient to determine the quantum operator $2 f$.

These investigations are carried out within the geometric quantization framework of Kostant and Souriau [11-13]; the pertinent features of this theory are summarized in the next section. Throughout this paper, we adhere to the notation and terminology of [12].

3 A constraint $f$ is first class if, for each constraint $f^{\prime}$, the Poisson bracket $\left\{f, f^{\prime}\right\}$ induced by $\omega$ vanishes when restricted to $M$. A constraint which is not first class is second class 


\section{Quantization Assumptions}

The supplementary structures on a $2 n$-dimensional symplectic manifold $(X, \omega)$ needed for geometric quantization are a prequantization, a polarization and a metaplectic frame bundle.

A prequantization of $(X, \omega)$ consists of a complex line bundle $L$ over $X$ with a connection $\nabla$ and an invariant Hermitian inner product such that

$$
\text { curvature } \nabla=-h^{-1} \omega,
$$

where $h$ is Planck's constant.

A polarization of $(X, \omega)$ is an involutive $n$-dimensional complex distribution $F$ on $X$ such that

$$
\omega \mid(F \times F)=0
$$

and $\operatorname{dim}(F \cap \bar{F})$ is constant, where $\bar{F}$ denotes the complex conjugate of $F$. A polarization $F$ defines two complex distributions $F \cap \bar{F}$ and $F+\bar{F}$ which are the complexifications of certain real distributions $D$ and $E$, respectively:

$$
F \cap \bar{F}=D^{\mathbb{C}} \text { and } F+\bar{F}=E^{\mathbb{C}} .
$$

Note that $D^{\perp}=E$, where " $\perp$ " denotes the symplectic orthogonal complement. ${ }^{4}$ Since $F$ is involutive $D$ is also, so that $D$ defines a foliation of $X$. We denote by $X / D$ the space of all integral manifolds of $D$ and by $\pi_{D}$ the canonical projection $X \rightarrow X / D$. A polarization $F$ is strongly admissible if $E$ is involutive, the spaces $X / D$ and $X / E$ of integral manifolds of $D$ and $E$, respectively, are (Hausdorff) quotient manifolds of $X$ and the canonical projection $\pi_{E D}: X / D \rightarrow X / E$ is a submersion. If $F$ is strongly admissible, then each fiber $P$ of $\pi_{E D}$ has a pseudoKähler structure such that $F \mid \pi_{D}{ }^{-1}(P)$ projects onto the distribution of antiholomorphic vector fields on $P[12]$.

A metaplectic structure on $(X, \omega)$ is given by a bundle of metaplectic frames, that is, a right principal $M p(n, \mathbb{R})$ bundle over $X$ doubly covering the symplectic frame bundle of $(X, \omega) .{ }^{5}$ The metaplectic structure is used to define the complex line bundle $\sqrt{ } \wedge{ }^{n} F$, the bundle of half-forms relative to $F$. This bundle has a canonically defined partial flat connection covering $F$.

We denote by $\mathscr{H}$ the quantum state space corresponding to the geometric quantization structures given above. Elements of $\mathscr{H}$ are sections of the complex line bundle $L \otimes \sqrt{ } \wedge^{n} F$ which are covariantly constant along $F$. If $F$ is strongly admissible then, equivalently, wave functions $\sigma \in \mathscr{H}$ are represented by sections of $L \otimes \sqrt{ } \wedge{ }^{n} F$ which are covariantly constant along $D$ and holomorphic along the fibers of $\pi_{E D}$. Such sections have supports contained in the subset $S$ of $X$ which is the union of those integral manifolds $A$ of $D$ for which the holonomy group of the induced flat connection in $\left(L \otimes \sqrt{ } \wedge^{n} F\right) \mid \Lambda$ is trivial. The set $S$ is called the BohrSommerfeld variety since it is locally determined by the generalized BohrSommerfeld conditions.

4 If $(V, \omega)$ is a symplectic vector space and $W \subseteq V$ is a linear subspace, then the symplectic orthogonal complement $W^{\perp}$ of $W$ in $V$ is the set of all $v \in V$ such that $\omega(v, w)=0$ for all $w \in W$

5 The metaplectic group $M p(n, \mathbb{R})$ is the double covering of the symplectic group $S p(n, \mathbb{R})$ 
Each integral manifold $\Lambda$ of $D$ has a canonically defined parallelization. Assuming that the polarization $F$ is strongly admissible and complete (in the sense that the leaves of $D$ are complete affine manifolds), it is possible $[20,21]$ to decompose the Bohr-Sommerfeld variety.

$$
S=\bigcup_{k=0}^{d} S_{k},
$$

where $d=\operatorname{dim} D$ and $S_{k}$ is the union of all those leaves of $D$ contained in $S$ which are affinely isomorphic to the cylinder $\mathbb{T}^{k} \times \mathbb{R}^{d-k}$. The dimension of each such submanifold $S_{k}$ is $2 n-k$.

\section{Coisotropic Imbeddings and Quantization}

Let $(M, \Omega)$ be a presymplectic manifold, and consider an imbedding of $M$ into a symplectic manifold $(X, \omega)$. As noted in the Introduction, inconsistencies may arise when one quantizes the imbedding $(M, \Omega) \rightarrow(X, \omega)$ if second class constraints are present. To circumvent this difficulty, Dirac [17] suggested replacing the Poisson bracket by a new bracket operation which is now known as a Dirac bracket $[14,19]$. The transition from a Poisson to a Dirac bracket corresponds to choosing a sympletic submanifold $Y$ of $X$ in such a way that $M$ is defined as a submanifold of $Y$ by the vanishing of first class constraints only. Thus, by introducing a Dirac bracket, one can effectively eliminate the second class constraints from the theory.

Dirac's local constructions suggest the existence of a certain class of imbeddings $(M, \Omega) \rightarrow(X, \omega)$ which will lead to consistent quantum dynamics. The fact that second class constraints cannot appear implies that such imbeddings must be "coisotropic." Specifically, a closed imbedding $j$ of a presymplectic manifold $(M, \Omega)$ into a (connected) symplectic manifold $(X, \omega)$ is coisotropic provided both $j^{*} \omega=\Omega$ and $\mathscr{T} M^{\perp} \subseteq \mathscr{T} j(\mathscr{T} M)$. The Poisson bracket on $(X, \omega)$ is, in this context, called a "Dirac bracket" [19].

For the purpose of quantization, it is important to determine if coisotropic imbeddings of a given presymplectic manifold exist. Furthermore, to ensure that the notion of Dirac bracket is not too arbitrary to be useful, it is necessary to classify all possible coisotropic imbeddings. The following theorem settles both of these issues.

Coisotropic Imbedding Theorem $[18,19]$ : Any presymplectic manifold $(M, \Omega)$ can be coisotropically imbedded in some symplectic manifold. Moreover, given any two such imbeddings $j_{1}:(M, \Omega) \rightarrow\left(X_{1}, \omega_{1}\right)$ and $j_{2}:(M, \Omega) \rightarrow\left(X_{2}, \omega_{2}\right)$, there exists a symplectomorphism $\psi$ from a neighborhood of $j_{1}(M)$ in $X_{1}$ onto a neighborhood of $j_{2}(M)$ in $X_{2}$ such that $j_{2}=\psi \circ j_{1}$.

The last part of this Theorem implies that a neighborhood of the coisotropic submanifold $M$ in a symplectic imbedding space $(X, \omega)$ is completely determined by $(M, \Omega)$ up to a symplectomorphism which reduces to the identity on $M$. In other words, the coisotropic imbedding $(M, \Omega) \rightarrow(X, \omega)$ is "locally unique."

As remarked upon earlier, this Theorem has the consequence that the classical 
symplectic dynamics of the system under consideration is effectively independent of the choice of coisotropic imbedding $(M, \Omega) \rightarrow(X, \omega)$. On the quantum level, one would hope that an analogous result holds. Unfortunately, even though the structure of a neighborhood of $M$ in $X$ is uniquely determined, $X$ can be quite arbitrary globally. Since the quantization process depends significantly upon the global topology and geometry of the imbedding space $(X, \omega)$, it is clear that one cannot expect the quantization of the system to be independent of the choice of $(X, \omega)$ in general. On the other hand, the above Theorem does imply that all quantization constructions which employ only (arbitrarily small) neighborhoods of $M$ in $X$ are insensitive to the choice of $(X, \omega)$.

One can nevertheless eliminate a substantial portion of the dependence of the quantization upon the choice of $(X, \omega)$ by restricting consideration to certain simple types of coisotropic imbeddings. For instance, it is always possible to choose the imbedding $M \rightarrow X$ in such a way that $X$ is a tubular neighborhood of the zero section of some vector bundle over $M .^{6}$ As an illustration of the usefulness of such imbeddings, consider the construction of the prequantization line bundle and the metaplectic frame bundle over the imbedding space $(X, \omega)$.

The existence and uniqueness of these structures depend upon the topology of $X$ and can be characterized cohomologically $[11,13]$. According to general principles, one would like these bundles to be determined by the topology of $M$ rather than that of $X$. This will in fact be the case if $X$ is taken to be a tubular neighborhood of $M$, since such an $X$ is homotopic to $M$. Since the choice of ambient space is to a certain extent arbitrary in any case, one loses no generality by restricting $X$ in this manner.

Thus it is always possible, by suitably restricting the class of allowable imbeddings, to characterize the prequantization and metaplectic structures solely in terms of the cohomology of $M$. Recent work of Vaisman [22] shows that this is also the case regarding the existence of (certain types of) polarizations of $(X, \omega)$. It remains to determine to what extent a similar result holds concerning the quantum representation space.

\section{Quantum Wave Functions}

Let the presymplectic manifold $(M, \Omega)$ be coisotropically imbedded in the connected symplectic manifold $(X, \omega)$ where, in view of the remarks at the end of the last section, it is assumed that $X$ is a tubular neighborhood of the zero section of some vector bundle over $M$. Let $\mathscr{H}$ denote the space of quantum states associated to $(X, \omega)$ by the geometric quantization procedure. In this section we study the dependence of $\mathscr{H}$ upon the choice of imbedding $M \rightarrow X$. Since the structure of $\mathscr{H}$ is very sensitive to the choice of polarization, so also is the extent to which the coisotropic submanifold $M$ determines $\mathscr{H}$.

For a completely general polarization $F$ we have the following fundamental result.

6 In particular, one could take this vector bundle to be the dual of the characteristic bundle of $(M, \Omega)$, cf. $[18-19]$ 
Proposition 1. If the Bohr-Sommerfeld variety $S$ is contained in $\pi_{D}^{-1}\left(\pi_{D}(M)\right)$, then each section $\sigma \in \mathscr{H}$ is uniquely determined by its restriction to $M$.

Proof. If $S \subseteq \pi_{D}{ }^{-1}\left(\pi_{D}(M)\right)$, then each leaf of $D$ contained in $S$ must intersect $M$. Since wave functions $\sigma \in \mathscr{H}$ are sections of $L \otimes \sqrt{ } \wedge{ }^{n} F$ covariantly constant along $F$ with supports contained in $S$, it follows that any $\sigma \in \mathscr{H}$ can be reconstructed from $\sigma \mid M$ by parallel transport along the leaves of $D$.

A polarization is real if $F=\bar{F}$. For such polarizations, the converse of Proposition 1 holds. Indeed, if each section $\sigma$ is determined by its restriction to $M$, then elements of $\mathscr{H}$ must have their supports in $\pi_{D}{ }^{-1}\left(\pi_{D}(M)\right)$; thus $S \subseteq \pi_{D}{ }^{-1}\left(\pi_{D}(M)\right)$. This proves

Proposition 2. Let $F$ be a real polarization and $\mathscr{H}$ the corresponding representation space. If each $\sigma \in \mathscr{H}$ is uniquely determined by its restriction to $M$, then the Bohr-Sommerfeld variety $S$ is contained in $\pi_{D}^{-1}\left(\pi_{D}(M)\right)$.

It should be noted that the reality condition on $F$ is essential. If $F$ is even partially complex (i.e., $\operatorname{dim} D<n$ ), then Proposition 2 can fail. ${ }^{7}$

Since Propositions 1 and 2 do not require strong admissibility, they can be used to study "wild" polarizations. To simplify matters, however, it is convenient to classify polarizations $F$ according to the nature of the intersection of $E$ with $\mathscr{T} M^{\perp}$. We assume that

$$
\operatorname{dim}\left(E \cap \mathscr{T} M^{\perp}\right)=\ell
$$

is constant on $M$. We begin with the transverse case $\ell=0$.

Theorem 1. Let $M$ be a compact submanifold of $(X, \omega)$ and let $F$ be a strongly admissible polarization of $(X, \omega)$ such that $E \cap \mathscr{T} M^{\perp}=\{0\}$. Then each section $\sigma \in \mathscr{H}$ is uniquely determined by its restriction to $M$.

Proof. We show that $\pi_{D}^{-1}\left(\pi_{D}(M)\right)$ is both open and closed in $X$. Since $X$ is assumed connected, the desired result follows from Proposition 1.

Since $E^{\perp}=D$, the assumption that $E$ is transverse to $\mathscr{T} M^{\perp}$ implies that

$$
D+\mathscr{T} M=\mathscr{T}_{M} X .
$$

Consequently, if $d=\operatorname{dim} D$,

$$
\operatorname{dim}[\mathscr{T} M /(D \cap \mathscr{T} M)]=2 n-d=\operatorname{dim}(X / D),
$$

so that $\pi_{D}(M)$, which is isomorphic to the space of integral manifolds of $D \cap \mathscr{T} M$, is open in $X / D$. Since $M$ is compact, so is $\pi_{D}(M)$ and, as $X / D$ is assumed Hausdorff, $\pi_{D}(M)$ is closed in $X / D$. The continuity of $\pi_{D}$ then implies that $\pi_{D}{ }^{-1}\left(\pi_{D}(M)\right)$ is a connected component of $X$.

The compactness hypothesis in this theorem can be dropped by means of the

7 Here is a counterexample to Proposition 2 in the case when $F$ is "totally" complex. Take $X=\mathbb{C}$, $\omega=-i d \bar{z} \wedge d z$ and let $F$ be spanned by the anti-holomorphic vector field $\partial / \partial \bar{z}$. Then each wave function $\sigma \in \mathscr{H}$ is of the form $\sigma=\psi \sigma_{0}$, where $\sigma_{0}$ is any trivialization of $L \otimes \sqrt{ } \wedge{ }^{1} F$ covariantly constant along $F$ and $\psi$ is holomorphic. If $M$ is any compact 1-dimensional submanifold of $\mathbb{C}$ then each holomorphic function on $\mathbb{C}$, and therefore each section in $\mathscr{H}$, is uniquely determined by its restriction to $M$. However, since $D=\{0\}, S=\mathbb{C}$ and $\pi_{D}^{-1}\left(\pi_{D}(M)\right)=M$ does not contain $S$. The authors are indebted to $I$. Singer for discussions regarding this point 
following construction. The essential use of compactness is to guarantee that every leaf of $D$ intersects the submanifold $M$. If $M$ is not compact, then there may exist integral manifolds of $D$ which do not meet $M$. However, since $X^{\prime}=\pi_{D}{ }^{-1}\left(\pi_{D}(M)\right)$ is an open submanifold of $X$, we may simply "throw away" such leaves by considering $M$ as being imbedded in $X^{\prime}$ rather than $X$. Clearly, $X^{\prime}$ is symplectic and inherits all necessary geometric quantization structures from $X$. In particular, the restriction of $F$ to $X^{\prime}$ is a polarization of $\left(X^{\prime}, \omega \mid X^{\prime}\right)$ and $\left(D \mid X^{\prime}\right)^{\mathbb{C}}=\left(F \mid X^{\prime}\right) \cap\left(\bar{F} \mid X^{\prime}\right)$. We may thus quantize $\left(X^{\prime}, \omega \mid X^{\prime}\right)$ rather than $(X, \omega)$. Then by construction every integral manifold of $D \mid X^{\prime}$ will intersect $M$ so that the conclusion of the theorem holds for covariantly constant sections of $\left(L \mid X^{\prime}\right) \otimes \sqrt{ } \wedge^{n}\left(F \mid X^{\prime}\right)$.

Now, let us turn to non-transverse polarizations. When $\ell \neq 0$ it is not usually true that each wave function is uniquely determined by its restriction to $M$. If, however, this is the case, then we obtain a topological restriction on the polarization.

Theorem 2. Let $F$ be a complete strongly admissible real polarization of $(X, \omega)$ such that $\pi_{D}: X \rightarrow X / D$ is a locally trivial fibration. If each section $\sigma \in \mathscr{H}$ is uniquely determined by its restriction to $M$, then the integral manifolds of $D$ are diffeomorphic to $\mathbb{T}^{k} \times \mathbb{R}^{n-k}$ with $k \geqq \ell$, where $\ell=\operatorname{dim}\left(D \cap \mathscr{T} M^{\perp}\right)$.

Proof. Since $\pi_{D}$ is a locally trivial fibration and $F$ is both real and complete, all integral manifolds of $D$ are diffeomorphic to $\mathbb{T}^{k} \times \mathbb{R}^{n-k}$ for some fixed $k, 0 \leqq k \leqq n$. Thus the Bohr-Sommerfeld variety $S=S_{k}$, where $\operatorname{dim} S_{k}=2 n-k$. Furthermore, by Proposition $2, S$ is contained in $\pi_{D}^{-1}\left(\pi_{D}(M)\right)$. Since

$$
\begin{aligned}
\operatorname{dim}\left[\pi_{D}^{-1}\left(\pi_{D}(M)\right)\right] & =\operatorname{dim}(D+\mathscr{T} M) \\
& =\operatorname{dim}\left(D \cap \mathscr{T} M^{\perp}\right)^{\perp} \\
& =2 n-\ell,
\end{aligned}
$$

the inclusion $S \subseteq \pi_{D}{ }^{-1}\left(\pi_{D}(M)\right)$ implies that $k \geqq \ell$.

As a special case, consider a complete real polarization $F$ such that $\pi_{D}$ is a locally trivial fibration and $\mathscr{T} M^{\perp} \subseteq D$, so that $\ell=\operatorname{codim} M$. Such a polarization would play an important role in comparing the quantization in the extended phase space $(X, \omega)$ with the quantization of the reduced phase space $(\bar{M}, \bar{\Omega})$, since $F \cap \mathscr{T} M$ projects via $\rho$ to a polarization of $(\bar{M}, \bar{\Omega})$. If each section $\sigma \in \mathscr{H}$ is uniquely determined by its restriction to $M$, then Theorem 2 implies that $\mathscr{T} M^{\perp}$ can be extended to an involutive isotropic distribution $K$ on $X$ such that the integral manifolds of $K$ are $k$-tori, where $k \geqq \operatorname{codim} M[20,21]$. This seems to be a fairly severe restriction.

\section{Quantum Operators}

Classical dynamical variables are smooth functions on the phase space $(X, \omega)$. Geometric quantization assigns to some classical observables $f$ quantum operators $2 f$ in a way which we now briefly describe. Let the observable $f$ generate a oneparameter group $\phi_{f}{ }^{t}$ of symplectomorphisms of $(X, \omega)$. Given a polarization $F$, the $\phi_{f}{ }^{t}$ induce a one-parameter family of unitary transformations, also denoted by $\phi_{f}{ }^{t}$, from the representation space $\mathscr{H}$ defined by $F$ to the representation spaces $\mathscr{H}_{t}$ corresponding to the polarizations $\mathscr{T} \phi_{f}{ }^{t}(F)$. Subject to certain regularity assump- 
tions, it is possible to define the Blattner-Kostant-Sternberg kernels

$$
\mathscr{K}_{t}: \mathscr{H} \times \mathscr{H}_{t} \rightarrow \mathbb{C} \text {. }
$$

For each $\sigma \in \mathscr{H}$ and $\sigma_{t} \in \mathscr{H}_{t}$, the value of $\mathscr{K}_{t}\left(\sigma, \sigma_{t}\right)$ is obtained by integrating a local concomitant $\delta\left(\sigma, \sigma_{t}\right)$ constructed from the sections $\sigma$ and $\sigma_{t}$ over the space of leaves of the distribution $D \cap \mathscr{T} \phi_{f}{ }^{t}(D)$, c.f. [12]. Let $\mathscr{U}_{t}: \mathscr{H}_{t} \rightarrow \mathscr{H}$ be the linear map defined by

$$
\mathscr{K}_{t}\left(\sigma, \sigma_{t}\right)=\left(\sigma \mid \mathscr{U}_{t}\left(\sigma_{t}\right)\right)
$$

where $(\cdot \mid \cdot)$ is the inner product on $\mathscr{H}$. The quantum operator $\mathscr{Q} f$ corresponding to $f$ is given by

$$
\mathscr{2} f[\sigma]=\left.i \hbar \frac{d}{d t}\left\{\left(\mathscr{U}_{t}^{\circ} \phi_{f}{ }^{t}\right) \sigma\right\}\right|_{t=0} .
$$

For a fixed polarization $F$ of $(X, \omega)$, the operator $2 f$ will in general depend upon both the global properties of $f$ on $X$ and the geometry and topology of the imbedding space $(X, \omega)$. On the other hand, the Coisotropic Imbedding Theorem implies that if the construction of $2 f$ employs only arbitrarily small neighborhoods of the coisotropic submanifold $M$ in $X$ then $2 f$ will be insensitive to both the largescale behavior of $f$ and the choice of imbedding $M \rightarrow X$. The aim of this section is to find the conditions under which it is possible to determine $2 f$ from the knowledge of $f$ in an arbitrarily small neighborhood of $M$.

It follows from Theorem 2 that, if $\ell=\operatorname{dim}\left(E \cap \mathscr{T} M^{\perp}\right) \neq 0$ and each $\sigma \in \mathscr{H}$ is uniquely determined by its restriction to $M, \mathscr{H}$ consists of discontinuous sections of $L \otimes \sqrt{ } \wedge^{n} F$ with supports specified by $k \geqq \ell$ Bohr-Sommerfeld conditions. Since the presence of Bohr-Sommerfeld conditions usually restricts the class of quantizable functions and, moreover, since the explicit construction of $2 f$ is quite complicated in these cases [12], we assume in what follows that $E \cap \mathscr{T} M^{\perp}=\{0\}$.

Theorem 3. Suppose that $M$ is a compact submanifold of $(X, \omega)$, and let $F$ be a sufficiently regular strongly admissible polarization of $(X, \omega)$ for which $E \cap \mathscr{T} M^{\perp}=\{0\}{ }^{8}$ Let $f \in C^{\infty}(X)$ be such that $2 f$ exists. If there exists an $\varepsilon>0$ such that, for each $t \in[0, \varepsilon), D_{t}=D \cap \mathscr{T} \phi_{f}{ }^{t}(D)$ is a distribution on $X$ satisfying

$$
D_{t}+\mathscr{T} M=\mathscr{T}_{M} X
$$

then, for each $\sigma \in \mathscr{H}, \mathscr{2} f[\sigma]$ can be determined by operations in an arbitrarily small neighborhood of $M$ in $X$.

Proof. By definition,

$$
(v \mid \mathscr{Q} f[\sigma])=\left.i \hbar \frac{d}{d t}\left[\mathscr{K}_{t}\left(v, \phi_{f}{ }^{t}(\sigma)\right)\right]\right|_{t=0} .
$$

It therefore suffices to show that, for each open set $U$ containing $M$, there exists

8 By "sufficiently regular" we mean a polarization for which the kernels $\mathscr{K}_{t}$ are defined and converge. See [12] for a detailed explanation 
$t_{U}>0$ such that $\mathscr{K}_{t}\left(v, \phi_{f}{ }^{t}(\sigma)\right)$ is determined solely by operations in $U$ for each $t \in\left[0, t_{U}\right)$.

Since $E \cap \mathscr{T} M^{\perp}=\{0\}$ Theorem 1 implies that both $\sigma$ and $v$ are uniquely determined by their restrictions to $M$. Hence $\phi_{f}{ }^{t}(\sigma) \in \mathscr{H}_{t}$ is uniquely determined by $\sigma \mid \phi_{f}{ }^{t}(M)$. Furthermore, since $M$ is compact, for each open set $U$ containing $M$ there exists $t_{U}>0$ such that $\phi_{f}{ }^{t}(M) \subset U$ for all $t \in\left[0, t_{U}\right)$.

Now

$$
\mathscr{K}_{t}\left(v, \phi_{f}{ }^{t}(\sigma)\right)=\int_{X / D_{t}} \delta\left(v, \phi_{f}{ }^{t}(\sigma)\right)
$$

where for each $t$ we assume that the space $X / D_{t}$ of integral manifolds of the distribution $D_{t}=D \cap \mathscr{T} \phi_{f}{ }^{t}(D)$ is a (Hausdorff) quotient manifold of $X$ with projection $\pi_{t}$. Mimicking the proof of Theorem 1 , the assumption that $D_{t}+\mathscr{T} M=\mathscr{T}_{M} X$ implies that $\pi_{t}$ maps $M$ onto $X / D_{t}$. Consequently, the integration appearing in the definition of the kernel $\mathscr{K}_{t}$ is actually over $\pi_{t}(M)$. Moreover, since the local concomitant $\delta\left(v, \phi_{f}{ }^{t}(\sigma)\right)$ depends only upon the sections $v$ and $\phi_{f}{ }^{t}(\sigma)$ it follows that, for each $t \in\left[0, t_{U}\right)$, the integral of $\delta\left(v, \phi_{f}{ }^{t}(\sigma)\right)$ over $X / D_{t}$ can be determined solely by the knowledge of $\sigma, v$ and $f$ in $U$, as required.

For a given polarization $F$ of $(X, \omega)$, there is a distinguished subclass of observables $f \in C^{\infty}(X)$ which "preserve" $F$ in the sense that $\mathscr{T} \phi_{f}{ }^{t}(F)=F$. Every such observable $f$ is quantizable [12] and the above expression for $\mathscr{2} f$ reduces to

$$
\mathscr{2} f[\sigma]=\left.i \hbar \frac{d}{d t}\left[\phi_{f}^{t}(\sigma)\right]\right|_{t=0}
$$

for all $\sigma \in \mathscr{H}$. If $F$ is chosen so that $E \cap \mathscr{T} M^{\perp}=\{0\}$ then, since $D_{t}=D$, the hypotheses of Theorem 3 are satisfied. Thus we have proven the following.

Corollary. Suppose that the polarization $F$ is such that $E$ is transverse to $\mathscr{T} M^{\perp}$. If $f$ is an observable which preserves $F$, then the corresponding quantum operator $2 f$ exists and is independent of the choice of coisotropic imbedding $(M, \Omega) \rightarrow(X, \omega)$.

As was the case with Theorem 1, the hypotheses of Theorem 3 can be weakened somewhat under certain circumstances. Specifically, if one restricts attention to those observables $f$ which both preserve $F$ and are "first class" (i.e., $v[f]=0$ for all $v \in \mathscr{T} M^{\perp}$ ), then the compactness assumption may be dropped. Indeed, note that compactness was used only twice in the proof of Theorem 3 . In the first instance, it guaranteed that the projection $\pi_{t}$ maps $M$ onto $X / D_{t}$ for each $t \in\left[0, t_{U}\right)$. If $M$ is not compact, this will not necessarily be the case. As in Sect. IV, however, one may view $M$ as being imbedded in

$$
X^{\prime}=\bigcap_{t \in\left[0, t_{U}\right)} \pi_{t}^{-1}\left(\pi_{t}(M)\right)
$$

rather than $X$-provided $X^{\prime}$ is an open submanifold of $X$ - for then by construction the projections $M \rightarrow X^{\prime} /\left(D_{t} \mid X^{\prime}\right)$ will be onto for each $t \in\left[0, t_{U}\right)$. In particular, if $f$ preserves $F$ then $D_{t}=D$ and $\pi_{t}=\pi_{D}$ are independent of $t$, and in this case $X^{\prime}=\pi_{D}{ }^{-1}\left(\pi_{D}(M)\right)$ is open in $X$. The second use of compactness was to ensure that $\phi_{f}{ }^{t}(M) \subset U$ for each $t \in\left[0, t_{U}\right)$. But this will always be the case if $f$ is first class, since the flow of any first class observable $f$ preserves $M$, i.e., $\phi_{f}{ }^{t}(M)=M$. 


\section{Discussion}

The present work is but a first step in understanding the quantization of a presymplectic dynamical system. We have shown that it is always possible to geometrically quantize a presymplectic manifold by coisotropically imbedding it in a symplectic manifold. Moreover, we have delineated the extent to which the quantization so obtained is independent of the specific choice of imbedding. But there are many problems that we have only touched upon, and others that we have not considered at all. In this section we briefly glance at some of these problems, with an eye to both their eventual resolution and future work.

Let $(M, \Omega)$ be coisotropically imbedded in the symplectic manifold $(X, \omega)$, and let $\mathscr{H}$ be the quantum state space associated to $(X, \omega)$ by the geometric quantization procedure. As discussed in the Introduction, the set $\mathscr{H}_{0}$ of dynamically admissible quantum states of the system consists of those $\sigma \in \mathscr{H}$ for which $2 f[\sigma]=0$ for all quantizable classical constraints $f$.

The first set of problems concerns the actual construction of the space $\mathscr{H}_{0}$. For instance, for $\sigma$ to belong to $\mathscr{H}_{0}$ is it necessary to require that $\mathscr{Q} f[\sigma]=0$ for every constraint $f$, or will it suffice to require this for a set of $\operatorname{codim} M$ functionally independent constraints $f$ ? How does one invariantly construct $\mathscr{H}_{0}$ if $M$ cannot be globally defined as a submanifold of $X$ by the vanishing of constraint functions? Furthermore, what if the classical constraints are not quantizable in the representation given by the polarization $F$ ?

It is possible to partially answer these questions in the following situation. Suppose that one can identify the coisotropic submanifold $M$ with the zero-level set of the momentum mapping $J$ associated with the Hamiltonian action of some Lie group $G$ on the symplectic manifold $(X, \omega)[1,15,16]$. That is, suppose

$$
M=\left\{x \in X \mid \hat{J}_{\xi}(x)=0 \text { for all } \xi \in \mathfrak{g}\right\},
$$

where $\mathfrak{g}$ is the Lie algebra of $G$, the momentum mapping $J: X \rightarrow \mathfrak{g}^{*}$ and

$$
\hat{J}_{\xi}(x)=\langle J(x), \xi\rangle \text {. }
$$

If the quantization map is linear, then to determine $\mathscr{H}_{0}$ it suffices to require that $2 \hat{J}_{\xi}[\sigma]=0$ for all $\xi$ belonging to a basis of $\mathfrak{g}$. Furthermore, if the polarization $F$ is $G$-invariant, then the constraints $\hat{J}_{\xi}$ are directly quantizable as first-order differential operators on $\mathscr{H}$. If, in addition, the polarization is such that $E \cap \mathscr{T} M^{\perp}=\{0\}$ then, since the $\hat{J}_{\xi}$ are first class, Theorem 3 and its corollaries imply that the operators $2 \widehat{J}_{\xi}$ will be defined independently of the choice of $(X, \omega)$. However, if $F$ is not $G$-invariant, then one must employ the Blattner-Kostant-Sternberg kernels in order to quantize the $\hat{J}_{\xi}$. In this case, the operators $2 \hat{J}_{\xi}$ may be more complicated-if they exist - and the conditions defining $\mathscr{H}_{0}$ may be more difficult to handle.

This special case notwithstanding, one would like to have an intrinsic means of constructing $\mathscr{H}_{0}$ which does not depend upon whether the constraints are globally defined and/or quantizable. More precisely, one would like to use the geometry of the imbedding $(M, \Omega) \rightarrow(X, \omega)$ to define a projection operator $\Pi$ on $\mathscr{H}$ in such a way that $\mathscr{H}_{0}=\Pi^{-1}\{0\}$. Operators of this type have been construc- 
ted by Guillemin and Sternberg [23] in the case when $X$ is a punctured cotangent bundle; perhaps their techniques will be of use in this context.

Another problem concerns the Hilbert space structure of $\mathscr{H}_{0}$. In principle, one knows how to make $\mathscr{H}$ into a Hilbert space; this involves only standard techniques [12]. However, if zero is in the continuous spectrum of the quantum constraints $\mathscr{2} f$ (e.g., if the group $G$ of the preceding discussion is noncompact), then $\mathscr{H}_{0}$ will not be a subspace of the Hilbert space $\mathscr{H}$ but rather will consist of distributional wave functions. The problem, then, is to make $\mathscr{H}_{0}$ itself into a Hilbert space. Although it is not clear how to do this in general, the work of Tulczyjew [24] enables one in some cases to introduce a scalar product on $\mathscr{H}_{0}$. Furthermore, the question arises as to whether the quantum operators $2 \mathrm{~g}$ for $g \in C^{\infty}(X)$ restrict to well-defined operators on $\mathscr{H}_{0} \cdot{ }^{9}$ This is in fact the case for first class observables since, if $g$ is first class, then $2 g$ commutes with every quantum constraint $2 f{ }^{10}$ If, however, $g$ is not first class, then it is not necessarily true that $2 g\left[\mathscr{H}_{0}\right] \subseteq \mathscr{H}_{0}$; in this case Tulczyjew's constructions lead to a family of operators representing $g$ on $\mathscr{H}_{0}$.

In addition to the quantization via imbedding technique studied here, there are (at least) two other ways of quantizing a presymplectic manifold $(M, \Omega)$ : quantize the reduced phase space $(\bar{M}, \bar{\Omega})$, and quantize subsequent to imposing a maximal gauge condition [cf. the Introduction]. Ultimately, of course, it is necessary to determine to what extent these various quantization procedures are equivalent; preliminary results have already been obtained along these lines [15]. One point of contact between these different techniques was noted in Sect. IV. Another occurs when one considers the quantization of a first class observable.

Suppose that the first class function $f$ is quantizable in the representation given by the polarization $F$; then necessarily $E_{t}=E+\mathscr{T} \phi_{f}{ }^{t}(E)$ is an involutive distribution on $X$ [12]. If in addition $D_{t}$ satisfies the hypothesis of Theorem 3 , then $\operatorname{dim} D_{t} \geqq \operatorname{codim} M$. In the case when equality holds, we claim that the distribution $G_{t}=E_{t} \cap \mathscr{T} M$ is involutive and complementary to $\mathscr{T} M^{\perp}$ in $\mathscr{T} M$. Indeed, since $D_{t} \oplus \mathscr{T} M=\mathscr{T}_{M} X$, it follows that $E_{t} \cap \mathscr{T} M^{\perp}=\{0\}$. Furthermore,

$$
\begin{aligned}
\operatorname{dim} G_{t} & =\operatorname{codim}\left(D_{t}+\mathscr{T} M^{\perp}\right) \\
& =\operatorname{dim} \mathscr{T} M-\operatorname{dim} \mathscr{T} M^{\perp}
\end{aligned}
$$

and the claim is proven. Thus, the integral manifolds of $G_{t}$ yield a foliation of $M$ by symplectic submanifolds transverse to $\mathscr{T} M^{\perp}$. If these submanifolds map diffeomorphically onto the reduced phase space $(\bar{M}, \bar{\Omega})$ under the canonical projection $M \rightarrow \bar{M}$, then we have a family of gauge conditions. The quantization of a presymplectic manifold admitting gauge conditions is studied in [15].

Acknowledgements. One of us (M. J. G.) would like to thank A. Weinstein and I. Singer for their helpful comments and the Mathematics Department of the University of California at Berkeley for its hospitality while part of this paper was being written.

9 One of the authors (M. J. G.) would like to thank P. Chernoff for bringing this point to his attention 10 This is, of course, provided that the "bracket goes to commutator" rule is valid for all observables concerned 


\section{References}

1. Abraham, R., Marsden, J. E.: Foundations of mechanics, 2nd edn. Reading, MA.: BenjaminCummings, 1978

2. Künzle, H. P. : J. Math. Phys. 13, 739-744 (1972)

3. Śniatycki, J., Tulczyjew, W. M.: Ann. Inst. H. Poincaré A15, 177-187 (1971)

4. Gotay, M. J., Nester, J. M., Hinds, G. : J. Math. Phys. 19, 2388-2399 (1978)

5. Gotay, M. J., Nester, J. M.: Group Theoretical Methods in Physics In: Lecture Notes in Physics, Beigelböck, W. et al. (eds.), Vol. 94, pp. 272-279 Berlin, New York: Heidelberg, Springer 1979.

6. Gotay, M. J., Nester, J. M. : Geometrical methods in mathematical physics, In: Lecture Notes in Mathematics, Kaiser, G., Marsden, J. E., (eds.), Vol. 775, pp. 78-104 Berlin, Heidelberg, New York: Springer-Verlag, 1980.

7. Gotay, M. J.: Presymplectic manifolds, geometric constraint theory and the Dirac-Bergmann theory of constraints, Dissertation, Univ. of Maryland Dept. of Physics and Astronomy Preprint No. 80-063 (1979)

8. Gotay, M. J., Nester, J. M.: Ann. Inst. H. Poincaré A30, 129-142 (1979)

9. Souriau, J.-M.: Structure des systèmes dynamiques. Paris: Dunod, 1970

10. Śniatycki, J., Tulczyjew, W. M.: Ann. Inst. H. Poincaré A16, 23-27 (1972)

11. Simms, D. J., Woodhouse, N. M. J. : Lectures on geometric quantization: Lecture Notes in Physics Vol. 53 Berlin, Heidelberg, New York: Springer 1976

12. Śniatycki, J.: Geometric quantization and quantum mechanics,: Applied Mathematical Sciences Series Vol. 30 Berlin, Heidelberg, New York: Springer 1980

13. Woodhouse, N. M. J. : Geometric quantization. Oxford: Clarendon Press 1980

14. Śniatycki, J. : Ann. Inst. H. Poincaré A20, 365-372 (1974)

15. Śniatycki, J. : On gauge, constraints and quantization, In: Lecture Notes in Mathematics (1981) (to appear)

16. Gotay, M. J., Isenberg, J. A., Marsden, J. E., Śniatycki, J., Yasskin, P.: On constraints, gauge transformations and momentum mappings in field theory, (in preparation 1981)

17. Dirac, P. A. M.: Lectures on quantum mechanics, Belfer Graduate School of Science Monograph Series Vol. 2 New York: Academic Press 1964

18. Gotay, M. J. : On coisotropic imbeddings of presymplectic manifolds. Proc. Am. Math. Soc. (1981) (to appear)

19. Gotay, M. J.: In: Geometric quantization, Gotay, M. J. (ed.) University of Calgary 1981

20. Śniatycki, J.: Int. J. Theor. Phys. 14, $277-288$ (1975)

21. Śniatycki, J., Toporowski, S., Int. J. Theor. Phys. 16, 615-633 (1977)

22. Vaisman, I. : The Bott obstruction to the existence of nice polarizations, University of Haifa Department of Mathematics Preprint 1980

23. Guillemin, V., Sternberg, S. : Am. J. Math. 101, 915-955 (1979)

24. Tulczyjew, W. M. : Bull. Acad. Pol. Sci., Sér. Sci. Math. Astr. Phys. 13, 329-333 (1965)

Communicated by S.-T. Yau

Received March 25, 1981 
